\title{
Influência da porosidade na condutividade térmica, resistência mecânica e coeficiente de permeabilidade do concreto permeável
}

Visando o desenvolvimento de tecnologias de adaptação e mitigação dos efeitos do aumento de temperatura no setor da construção civil, de modo a promove melhor desempenho térmico-mecânico, esse estudo teve por objetivo avaliar a influência da porosidade na condutividade térmica do concreto permeável, avaliando sua resistência mecânica e coeficiente de permeabilidade. O concreto permeável foi dosado pelo método de Nguyen et al. (2014) com índices de vazios de 15, 20, 25 e 30\%. Determinou-se o coeficiente de permeabilidade, a resistência à compressão e a tração na flexão pelas normas técnicas brasileiras e sua massa específica, porosidade total e condutividade térmica pelas normas internacionais. A análise estatística dos resultados mostrou que somente a resistência à tração na flexão não apresenta diferença significativa com a variação da porosidade. A porosidade total do concreto permeável foi de $23,1,28,9$, 33,3 e 34,0\%, respectivamente aos índices de vazios utilizados como parâmetro de entrada no método de dosagem de 15, 20, 25 e 30\%, cujas massas específicas variaram, respectivamente, de 2153 a $1857 \mathrm{~kg} \cdot \mathrm{m}-3$. Os dois traços de menores porosidades atingiram resistência à compressão acima de $20 \mathrm{MPa}$, e a resistência à tração na flexão dos quatro traços ficou acima de 4,0 MPa. O coeficiente de permeabilidade médio dos concretos permeáveis assumiu valores entre 8,2 e 21,8 m.s-1, A condutividade térmica e a porosidade total possuem uma relação inversamente linear com ótimo ajuste e seus valores médios diminuíram de 0,55 , a 0,44 W.m-1. K-1 conforme a porosidade total aumentou de 23,1 para 34,0\%. Diante do exposto considera-se que o concreto permeável pode ser aplicado na construção civil como material alternativo para fins de melhoria do conforto térmico, visto sua condutividade térmica, coeficiente de permeabilidade e bom desempenho mecânico, remetendo-se ao traço de melhor desempenho, com índice de vazios de $20 \%$ e porosidade total de $23,1 \%$.

Palavras-chave: Concreto permeável; Porosidade; Condutividade térmica; Permeabilidade; Resistência mecânica.

\section{Influence of porosity on thermal conductivity, mechanical strength and permeability coefficient of pervious concrete}

Aiming at the development of technologies for adaptation and mitigation of the effects of temperature increase in the civil construction sector, in order to promote better thermal-mechanical performance, this study aimed to evaluate the influence of porosity on the thermal conductivity of pervious concrete evaluating its mechanical resistance and permeability coefficient. Pervious concrete was dosed by the method of Nguyen et al. (2014) with void rates of 15 , 20, 25 and $30 \%$. The permeability coefficient, the compressive strength and the flexural tensile strength were determined by Brazilian technical standards and their specific mass, total porosity and thermal conductivity by international standards. The statistical analysis of the results showed that only the tensile strength in flexion does not present a significant difference with the variation in porosity. The total porosity of the pervious concrete was $23.1,28.9,33.3$ and $34.0 \%$, respectively to the void indices used as an input parameter in the dosing method of $15,20,25$ and $30 \%$, whose specific masses ranged, respectively, from 2153 to $1857 \mathrm{~kg} . \mathrm{m}-3$. The two lower porosity strokes reached compressive strength above $20 \mathrm{MPa}$, and the tensile strength in flexion of the four strokes was above 4.0 $\mathrm{MPa}$. The average permeability coefficient of pervious concretes assumed values between 8.2 and $21.8 \mathrm{~ms}-1$. The thermal conductivity and total porosity have an inversely linear relationship with excellent adjustment and their average values decreased from 0.55 to $0.44 \mathrm{Wm}-1$. $\mathrm{K}-1$ as the total porosity increased from 23.1 to $34.0 \%$. In view of the above, it is considered that pervious concrete can be applied in civil construction as an alternative material for the purpose of improving thermal comfort, given its thermal conductivity, permeability coefficient and good mechanical performance, referring to the best performance feature, with voids index of $20 \%$ and total porosity of $23.1 \%$.

Keywords: Pervious concrete; Porosity; Thermal conductivity; Permeability; Mechanical resistance.

Topic: Desenvolvimento, Sustentabilidade e Meio Ambiente

Reviewed anonymously in the process of blind peer

\section{Matheus Oliveira Ramos}

Universidade Regional de Blumenau, Brasil

matheus.engcivil13@gmail.com

\section{Ivone Gohr Pinheiro (iD)}

Universidade Regional de Blumenau, Brasil http://lattes.cnpq.br/6522200645618540 http://orcid.org/0000-0002-3552-4352 ivonegp@furb.br

Leondiniz Gomes de Sousa Junior Universidade Regional de Blumenau, Brasil http://lattes.cnpq.br/9905871412046950 http://orcid.org/0000-0002-8095-516X leonjuninhoceulp@gmail.com
Received: 20/04/2021

Approved: 21/05/2021

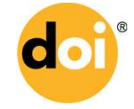

DOI: 10.6008/CBPC2179-6858.2021.005.0041
Referencing this:

RAMOS, M. O.; PINHEIRO, I. G.; SOUZA JUNIOR, L. G.. Influência da porosidade na condutividade térmica, resistência mecânica e coeficiente de permeabilidade do concreto permeável. Revista lbero Americana de Ciências Ambientais, v.12, n.5, p.515-528, 2021. DOI: http://doi.org/10.6008/CBPC2179-6858.2021.005.0041 


\section{INTRODUÇÃO}

O Painel Brasileiro de Mudanças Climáticas (PBMC) identificou que o clima do Brasil ficará entre 1 e 6 ํ C mais quente até 2100 , em relação às temperaturas registradas no final do século passado (PBMC, 2013). No contexto urbano, além dos efeitos das alterações climáticas locais, tem-se um microclima complexo e mais sensível as mudanças. Como exemplo, podem-se citar as ilhas de calor (WU et al., 2017). Nesse cenário, faz-se importante o desenvolvimento de tecnologias de adaptação e mitigação dos efeitos do aumento da temperatura global e local por parte do setor da construção civil, de modo a promover um desempenho térmico adequado das construções, frente a estas alterações climáticas (GALUSIC, 2019).

Uma das causas das ilhas de calor destacada por Gartland (2010) é a utilização de materiais impermeáveis e estanques, pois eles reduzem a umidade disponível para dissipação do calor. Outro fator é a configuração urbana tipo cânion que, quando combinada com revestimentos e pavimentos escuros, absorve e retém ainda mais a energia solar. Menciona-se ainda o calor produzido pela humanidade, a diminuição da velocidade do vento e a poluição do ar como causas da formação das ilhas de calor.

Pavimentos impermeáveis são conhecidos como um dos maiores contribuintes para as ilhas de calor, pois cobrem grande parte do solo urbano. Esses pavimentos podem aumentar as temperaturas locais, devido ao armazenamento de calor nos materiais utilizados, e podem reduzir o esfriamento por evaporação sobre as superfícies (WU et al., 2017).

Uma das formas de prevenção e atenuação desse fenômeno é a utilização de pavimentos permeáveis (EPA, 2008) que além de ser considerado um importante sistema de drenagem urbana sustentável (KIA et al., 2017; RAIMBAULT et al., 2002), se destacam promovendo outros benefícios ambientais, tais como a redução dos efeitos de ilhas de calor nas cidades e a diminuição dos ruídos relacionados à interação pneu-pavimento (HASELBACH et al., 2015; CURVO, 2017).

Os vazios interligados e a alta porosidade no concreto permeável se dão pela mistura de água e cimento, de tal forma a criar uma pasta para revestir os agregados, com a mínima ou nula presença de agregado miúdo, o que impacta diretamente em um maior coeficiente de permeabilidade e redução dos efeitos de ilhas de calor (CURVO, 2017). Os poros no interior do concreto permeável exibem boas propriedades térmicas e têm a capacidade de absorver a energia sonora através da fricção interna e, por conseguinte, podem ser utilizados como barreiras acústicas rodoviárias e materiais de isolamento térmico em edifícios (ARENAS et al., 2013).

Há uma gama de benefícios ambientais e socioeconômicos em edificações com bom desempenho térmico, como resultado da adoção de um comportamento de utilização eficiente de energia. Ambientalmente, os benefícios incluem a redução das emissões de gases de efeito estufa e redução da dependência de combustíveis fósseis não renováveis. Em uma perspectiva socioeconômica, edificações com bom desempenho térmico trazem benefícios ao bem-estar das pessoas e ainda podem reduzir os gastos com energia (SALAO, 2013; KALYANI et al., 2012).

A transferência de calor entre o interior de um edifício e o ambiente ao ar livre depende do desempenho térmico do edifício, devendo-se para isso, utilizar materiais com bom isolamento térmico, 
sendo o concreto permeável uma possível alternativa (SANTAMOURIS, 2013; NEMATOLLAHI, 2017).

A maior parte de consumo de energia em edifícios está associada com o aquecimento e a refrigeração (NEMATOLLAHI, 2017). Esta demanda de energia pode ser significativamente reduzida pelo uso de materiais de construção com menor condutividade térmica, proporcionando melhor isolamento térmico. Dessa forma, ao utilizar um material com baixa condutividade térmica em um edifício, contribuirse-á para a sustentabilidade, pois as necessidades energéticas na fase de utilização do edifício serão reduzidas (NEMATOLLAHI, 2017; WANDERLEY, 2018).

A escolha dos materiais construtivos está relacionada principalmente aos aspectos econômicos, de resistência mecânica e segurança, deixando muitas vezes o aspecto de desempenho térmico para um plano secundário. Os custos de aquecimento, em tempos frios, e refrigeração, em tempos quentes, são elevados. Um dos grandes desafios é o de melhorar o desempenho térmico dos edifícios, considerando, ainda a crescente ênfase social dada às questões ambientais (MATI-BAOUCHE et al., 2014).

Algumas propriedades que são imprescindíveis para caracterizar um material isolante térmico são a condutividade térmica, a resistividade térmica, o calor específico e a capacidade térmica (SOUZA, 2017). Os materiais isolantes térmicos apresentam baixa densidade, o que implica em baixa condutividade térmica e alta resistência térmica devido à alta porosidade. Portanto, quanto maior a porosidade dos materiais, maior vai ser a capacidade de impedir a transferência de calor (BEZERRA, 2003; ZHANG et al., 2015). A NBR 15220 (ABNT, 2005) define a condutividade térmica como a propriedade física de um material homogêneo e isótropo, no qual se verifica um fluxo de calor constante quando submetido a certo gradiente de temperatura.

O concreto permeável exibe boas características térmicas devido à grande quantidade de poros vazios no seu interior, e, por conseguinte, pode ser uma opção de material a ser utilizado no isolamento térmico de edificações (ARENAS et al., 2013). O concreto permeável apresenta condutividade térmica mais baixa que os blocos de concreto vazado e tijolos cerâmicos de oito furos usualmente utilizados na construção civil, podendo dessa forma ser utilizado nas edificações para fins de melhoria no conforto térmico (CHEN et al., 2019; NASSARI et al., 2017; CHINDAPRASIRT et al., 2014).

O concreto convencional possui uma condutividade térmica de $1,75 \mathrm{~W} \cdot \mathrm{m}^{-1} \cdot \mathrm{K}^{-1}$, enquanto a da argila expandida é de $0,16 \mathrm{~W} \cdot \mathrm{m}^{-1} \cdot \mathrm{K}^{-1}$ (ABNT, 2005). Blocos de concreto vazado, de $14 \times 19 \times 39 \mathrm{~cm}$ possuem condutividade térmica de $1,55 \mathrm{~W} \cdot \mathrm{m}^{-1} \cdot \mathrm{K}^{-1}$ (SOUZA, 2017) e blocos cerâmicos de oito furos, de $9 \times 19 \times 19 \mathrm{~cm}$, uma condutividade térmica de 0,9 W. $\mathrm{m}^{-1} \cdot \mathrm{K}^{-1}$ (GOMES, 2015). Em consequência do exposto, este trabalho teve como objetivo avaliar a influência da porosidade na condutividade térmica do concreto permeável, relacionando-a com as propriedades hidráulicas e mecânicas do concreto, considerando o fato de que é desejável um desempenho mínimo do concreto permeável no quesito resistência mecânica e coeficiente de permeabilidade.

\section{METODOLOGIA}

O concreto permeável foi dosado pelo método de Nguyen et al. (2014) e para sua confecção 
utilizou-se o cimento Portland CP V ARI, agregado graúdo, do tipo brita 0, como agregado miúdo, a areia grossa e uma relação água/cimento $(\mathrm{a} / \mathrm{c})$ de 0,32. O agregado miúdo correspondeu a 6,5\% do total de agregados, visto que essa quantidade proporciona uma importante melhoria da resistência mecânica do concreto permeável, sem comprometer sua porosidade (NGUYEN et al., 2014).

O agregado graúdo teve determinada sua massa específica, massa específica aparente e absorção de água conforme a NBR NM 53 (ABNT, 2009), distribuição granulométrica segundo a NBR NM 248 (ABNT, 2003) e massa unitária compactada e volume de vazios conforme a NBR NM 45 (ABNT, 2006). Para determinação da massa específica do agregado miúdo foi utilizada a NBR NM 52 (ABNT, 2009), para determinação da absorção de água a NBR NM 30 (ABNT, 2001) e o ensaio para determinação da distribuição granulométrica foi realizado conforme a NBR NM 248 (ABNT, 2003).

A curva granulométrica do agregado graúdo e do miúdo (Figura 1A e 1B) permite de constatar que eles se encontram dentro dos limites propostos pela norma NBR 7211 (ABNT, 2005), e o resultado dos outros ensaios (Tabela 1) foram utilizados para a determinação dos traços do concreto permeável.

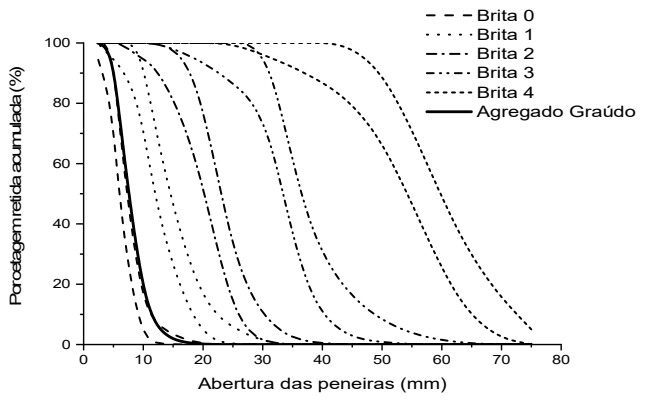

A)

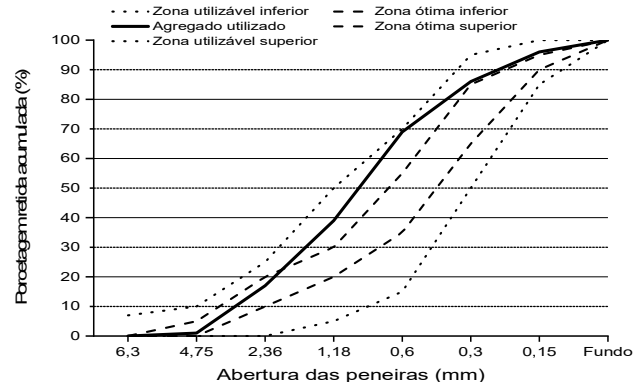

B)

Figura 1: Curva granulométrica do agregado comparada com limites da NBR 7211. A) agregado graúdo, B) agregado miúdo. Fonte: ABNT (2005).

A relação a/c foi obtida através do teste de "drenagem da pasta" descrito no método de Nguyen et al. (2014), que busca a relação a/c ótima, que foi de 0,32. O método possui como parâmetro de entrada o índice de vazios, tendo sido então contemplados quatro valores, $15 ; 20 ; 25$; e 30\%, cujos corpos de prova foram denominados de CP15, CP20, CP25 e CP30, respectivamente. A partir dos dados de caracterização dos agregados e com o resultado do teste de drenagem da pasta, foi possível calcular o traço, determinando-se o consumo de material para o concreto permeável (Tabela 1).

Tabela 1: Consumo de material e massa específica teórica do concreto permeável com diferentes índices de vazios.

\begin{tabular}{|c|c|c|c|c|c|c|c|}
\hline Traço & Índice de vazios & Cimento & Água & Brita & Areia & $a / c$ & Massa específica \\
\hline & (\%) & \multicolumn{5}{|c|}{ - } & $\mathrm{kg} \cdot \mathrm{m}^{-3}$ \\
\hline CP15 & 15 & 372 & 119 & 1628 & 101 & 0,32 & 2220 \\
\hline CP20 & 20 & 350 & 112 & 1532 & 95 & 0,32 & 2089 \\
\hline CP25 & 25 & 328 & 105 & 1436 & 89 & 0,32 & 1959 \\
\hline CP30 & 30 & 306 & 98 & 1341 & 83 & 0,32 & 1828 \\
\hline
\end{tabular}

A concretagem contemplou a execução de três placas de $350 \times 350 \times 100 \mathrm{~mm}$ e quatro placas de

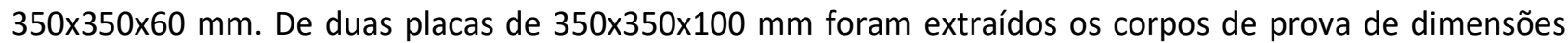
padrões de 100×100×350 mm para os ensaios de resistência a tração na flexão. Da outra placa de 


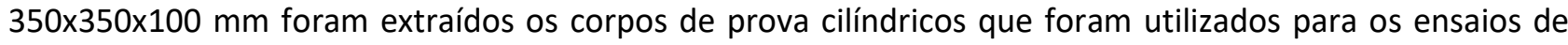
porosidade, massa específica e resistência à compressão. É importante ressaltar que sobre esses testemunhos cilíndricos, com dimensões de 50×100 mm, foram aplicadas as recomendações da norma NBR 7680 (ABNT, 2015) devido a sua redução de tamanho. Os corpos de prova foram extraídos em sextuplicata, enquanto as quatro placas de $350 \times 350 \times 60 \mathrm{~mm}$ foram reduzidas para as dimensões de $200 \times 200 \times 60 \mathrm{~mm}$ para serem submetidas ao ensaio de condutividade térmica (Quadro 1).

Quadro 1: Quantitativo dos corpos de provas, ensaios e normas utilizadas

\begin{tabular}{|c|c|c|c|c|c|}
\hline $\begin{array}{c}\text { DIMENSÕES DAS } \\
\text { PLACAS (mm) }\end{array}$ & $\begin{array}{c}\text { QUANT. DE } \\
\text { PLACAS }\end{array}$ & $\begin{array}{c}\text { DIMENSÕES DOS } \\
\text { CORPOS DE PROVA } \\
\text { (mm) }\end{array}$ & $\begin{array}{c}\text { QUANT. DE } \\
\text { CORPOS DE } \\
\text { PROVA }\end{array}$ & ENSAIO & NORMA \\
\hline $350 \times 350 \times 100$ & 2 & $\begin{array}{c}100 \times 100 \times 350 \\
\text { (prismático) }\end{array}$ & 6 & Tração na flexão & $\begin{array}{c}\text { NBR 12142 } \\
\text { (ABNT, 2010) }\end{array}$ \\
\hline $350 \times 350 \times 100$ & 1 & $50 \times 100$ (cilíndrico) & 6 & $\begin{array}{c}\text { NBR 5739 } \\
\text { (ABNT, 2018) } \\
\text { pesistência à compressão e } \\
\text { poridade e massa específica }\end{array}$ & $\begin{array}{c}\text { ASTM C1754 } \\
\text { (ASTM, 2012) }\end{array}$ \\
\hline $350 \times 350 \times 60$ & 4 & $200 \times 200 \times 60$ (placa) & 4 & $\begin{array}{c}\text { NBR 16416 } \\
\text { Coeficiente de permeabilidade } \\
\text { e condutividade térmica }\end{array}$ & $\begin{array}{c}\text { (ABNT, 2015) } \\
\text { e } \\
\text { ASTM C518 } \\
\text { (ASTM, 2017) }\end{array}$ \\
\hline
\end{tabular}

É importante ressaltar que todos os ensaios, com exceção de condutividade térmica, que foi realizado com o concreto permeável com 90 dias de idade, foram realizados após 28 dias de cura submersa em água. Antes de iniciar o ensaio de condutividade térmica as placas ficaram em estufa por $24 \mathrm{~h}$ com temperatura constante de $90^{\circ} \mathrm{C}$ para garantir que a umidade fosse nula, evitando, assim, que a determinação da condutividade térmica sofresse alteração conforme recomendação dos autores Chindaprasirt et al. (2014) e Nassiri et al. (2017). O equipamento utilizado foi o LaserComp, modelo FOX 304 , cuja precisão de temperatura é $\pm 0,01^{\circ} \mathrm{C}$ e de condutividade térmica é de $\pm 2 \%$. A execução dos corpos de prova de concreto permeável envolve um fator muito importante, a compactação. Foi através da variação da energia de compactação que foi possível atingir o índice de vazio imposto teoricamente na determinação do traço.

Quando da execução dos corpos de prova, a determinação da energia de compactação foi realizada de forma a se reproduzir no corpo de prova moldado a mesma massa específica teórica do traço. Determinou-se, então, a massa de concreto permeável necessária correspondente ao volume de cada uma das placas, sendo que a compactação foi realizada em mesa vibratória, com diferentes tempos de compactação a fim de atingir o índice de vazios teórico adotado (Tabela 2). No caso do CP30 não foi necessário vibrar, visto que sua massa é bem reduzida devido ao elevado índice de vazios.

Tabela 2: Massa de concreto permeável por traço $(\mathrm{kg})$ e por tipo de placa e tempo de compactação

\begin{tabular}{|c|c|c|c|}
\hline TRAÇO & PLACA DE 350x350x100 (mm) & PLACA DE $350 \times 350 \times 60(\mathrm{~mm})$ & $\begin{array}{l}\text { TEMPO DE COMPACTAÇÃO } \\
(\mathrm{s})\end{array}$ \\
\hline CP15 & 27,2 & 16,32 & 16 \\
\hline $\mathrm{CP} 20$ & 25,6 & 15,36 & 10 \\
\hline $\mathrm{CP} 25$ & 24 & 14,4 & 6 \\
\hline CP30 & 22,4 & 13,44 & 0 \\
\hline
\end{tabular}


Os resultados de cada grandeza avaliada foram submetidos a uma análise estatística através de teste de variância (ANOVA), com intervalo de confiança de 95\%, seguidos do teste de Tukey.

\section{RESULTADOS E DISCUSSÃO}

Os resultados são discutidos abordando-se primeiramente o coeficiente de permeabilidade, massa específica, porosidade e a resistência mecânica dos concretos permeáveis, a luz dos padrões normativos e literatura, para caracterizar o concreto permeável e depois discutir a condutividade térmica.

O coeficiente de permeabilidade dos concretos permeáveis apresentou uma grande variação em função do índice de vazios adotado no método de dosagem, variando de um valor mínimo de 8,03 ao máximo de $21,84 \mathrm{~mm} . \mathrm{s}^{-1}$, respectivamente ao traço de 15 e $30 \%$ de índice de vazios, com valor médio entre 8,24 e $21,80 \mathrm{~mm} . \mathrm{s}^{-1}$ (Figura 2). O menor coeficiente de permeabilidade, 8,03 mm.s ${ }^{-1}$, para o traço CP15, é ainda consideravelmente maior do que o mínimo segundo a NBR 16416 (ABNT, 2015), de 1 mm.s ${ }^{-1}$, e os resultados se encontram dentro da faixa de valores encontrados na literatura, entre 0,25 e $47,70 \mathrm{~mm} . \mathrm{s}^{-1} \mathrm{de}$ acordo com o estudo de revisão realizado por Zhong et al. (2015).

A análise estatística mostrou diferença significativa do coeficiente de permeabilidade com a variação de porosidade, havendo diferença significativa entre os traços CP15 e CP25, com o traço CP30 apresentando diferença significativa em relação a todos os demais tratamentos, não havendo, porém, diferença significativa entre os traços CP15 e CP20, e entre os traços CP20 e CP25.

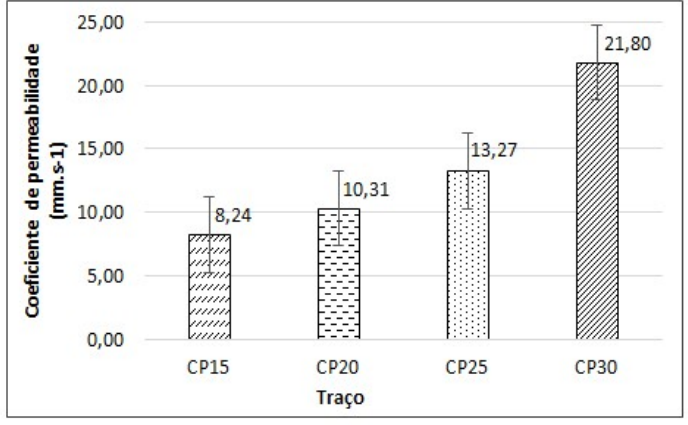

A)

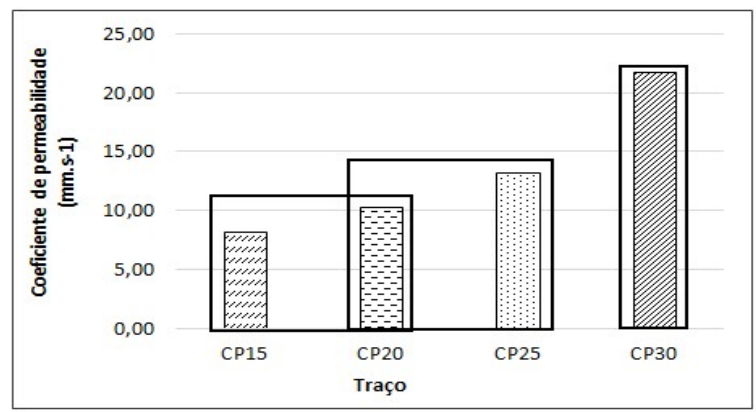

B)

Figura 2: A) Coeficiente de permeabilidade médio dos concretos permeáveis, B) Comparação entre as médias do coeficiente de permeabilidade dos concretos permeáveis

Onde: $\mathrm{CP} 15$ = Corpo de prova com índice de vazios de 15\%; CP20 = Corpo de prova com índice de vazios de $20 \%$; $\mathrm{CP} 25$ = Corpo de prova com índice de vazios de 25\%; CP30 = Corpo de prova com índice de vazios de 30\%

A porosidade dos concretos permeáveis assumiu valores médios entre 23,1 e $34,0 \%$ respectivamente ao traço CP15 e CP30 (Figura 3), observando-se que se trata da porosidade total, uma vez que foi obtida por meio da ASTM C1754 (ASTM, 2012).

Observa-se que os traços CP15 e CP20 apresentaram diferença significativa entre si e entre os demais tratamentos, no entanto, houve um agrupamento entre os traços CP25 e CP30, não apresentando diferença significativa entre si. O fato de que os traços CP25 e CP30 não apresentam diferença significativa entre si, pode ser devido à constatação de que independentemente do índice de vazios determinado como parâmetro de entrada do método de dosagem do método, existe um limite físico de porosidade que o concreto permeável pode atingir, ou seja, conforme se aumenta o índice de vazios (teórico na dosagem), a 
tendência da porosidade total (e do índice de vazios) do concreto permeável executado tende a um valor máximo, como para os índices de vazios acima de 25\%. De acordo com os autores Kia et al. (2017) e Lian et al. (2011), configurações de porosidades acima de $25 \%$ já são consideradas altas porosidades.

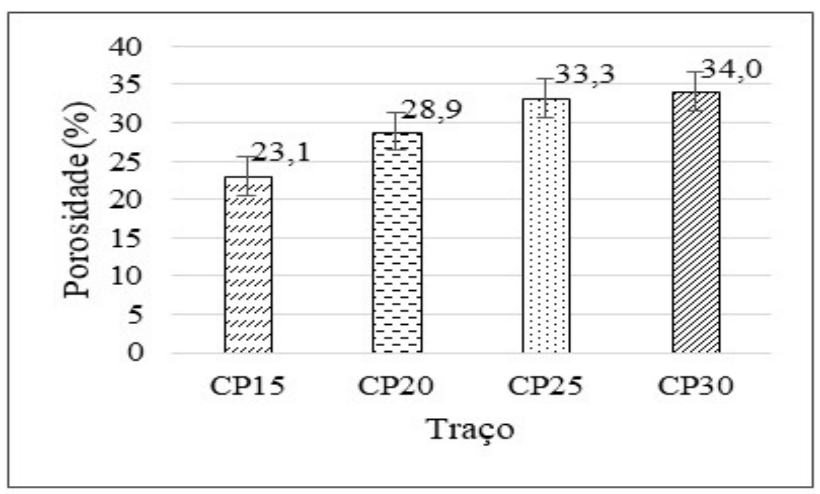

A)

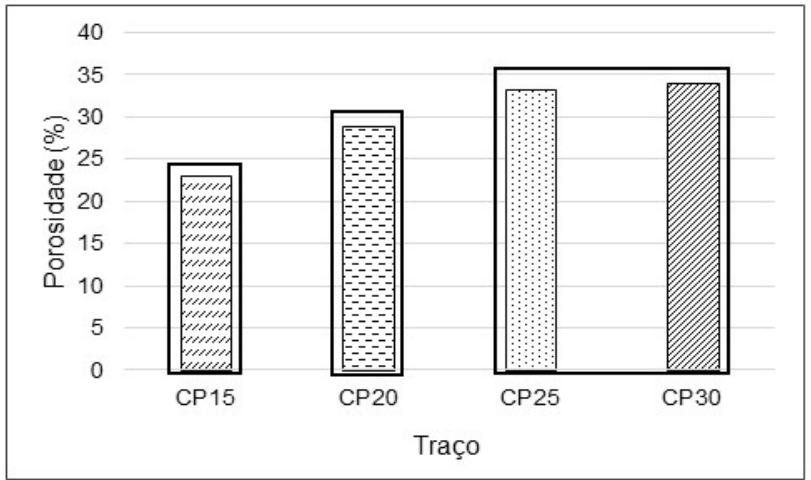

B)

Figura 3: A) Porosidade total, média dos concretos permeáveis, B) Comparação das médias de porosidade Onde: $\mathrm{CP} 15$ = Corpo de prova com índice de vazios de 15\%; CP20 = Corpo de prova com índice de vazios de $20 \%$; CP25 = Corpo de prova com índice de vazios de 25\%; CP30 = Corpo de prova com índice de vazios de 30\%

Os autores Zhong et al. (2016) sugerem que o sistema de poros no concreto permeável deveria ser diferenciado como porosidade efetiva e porosidade total, sendo que a primeira consiste na proporção do volume de poros acessíveis à agua em relação ao volume total do material, medida intrínseca à propriedade hidráulica, enquanto que a segunda consiste na relação entre volume total de poros, ou seja, volume de poros acessíveis e poros não acessíveis à água, e o volume total de material, medida intrínseca a resistência mecânica do concreto permeável. Ainda de acordo com os autores supracitados, o volume de poros não acessíveis pode constituído pelos poros formados no espaço entre os agregados envoltos pela pasta, não interligados entre si, os poros dos agregados e os poros da pasta.

De acordo com Nguyen et al. (2014), para a obtenção da porosidade total do concreto permeável, quatro fatores são determinantes, o índice de vazios, a porosidade dos agregados, a porosidade da pasta e a porosidade da zona de transição. Esses autores levam em conta para determinar a porosidade acessível à água somente o índice de vazios e a porosidade da pasta. Levando-se em conta Zhong et al. (2016) e Nguyen et al. (2014) pode-se considerar que a porosidade total determinada é maior do que o índice de vazios devido aos poros não acessíveis à água, mas também devido à porosidade da pasta.

A variação entre o índice de vazios inicialmente determinados e a porosidade total obtida, é de aproximadamente $8 \%$, para os traços CP15, CP20 e CP25 (Tabela 3). No que diz respeito ao traço CP30, a variação observada foi de apenas $4 \%$, porém é importante ressaltar a questão do limite máximo de porosidade que está condicionado à acomodação do material ao espaço do molde sem nenhuma compactação, condição que ocorreu para o corpo de prova com índice de vazios de $30 \%$.

Tabela 3: Diferença entre o índice de vazios adotado e a porosidade total determinada pela ASTM C1754 (ASTM,

\begin{tabular}{cccc}
\multicolumn{5}{c}{ 2012) } & Diferença (\%) \\
\hline Traço & Índice de vazios (\%) & Porosidade total (\%) & 8,1 \\
CP15 & 15 & 23,1 & 8,8 \\
CP20 & 20 & 28,9 & 8,3 \\
CP25 & 25 & 33,3 & 4,1 \\
CP30 & 30 & 34,0 & 4 \\
\hline
\end{tabular}


No que diz respeito à massa específica dos concretos permeáveis, os resultados médios variaram de 2153 a 1857 kg.m-3 (Figura 4). Na literatura, os valores de massa específica de concretos permeáveis se encontram na ordem de 1600 a 2200 kg.m-3 de acordo com o estudo de revisão realizado por Tennis et al. (2004) e conforme a NBR 16416 (ABNT, 2015), para concreto permeável moldado no local, o valor mínimo de massa específica deve ser de 1600 kg.m-3, portanto os resultados encontrados respondem a estes limites. Observa-se que tanto o traço CP15, como o traço CP20, apresenta diferença significativa em relação a todos os outros tratamentos, porém, há um agrupamento entre os traços CP25 e CP30, não havendo diferença significativa entre si (Figura 4), reproduzindo o comportamento da porosidade dada a correlação entre as grandezas através da energia de compactação imposta.

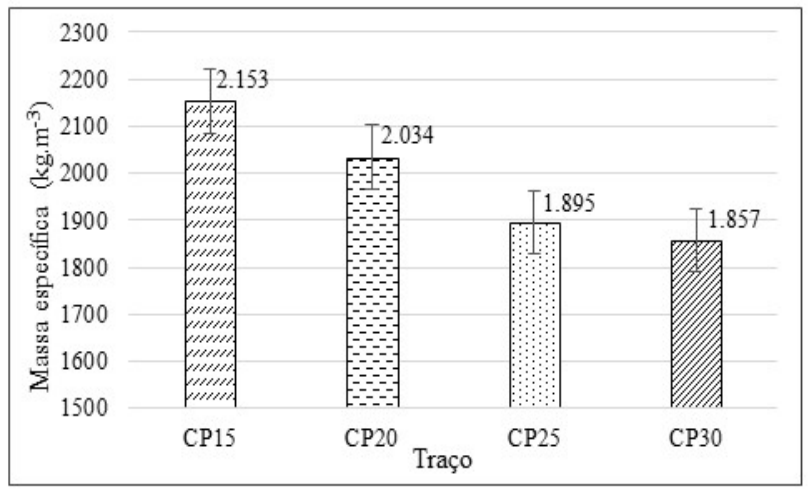

A)

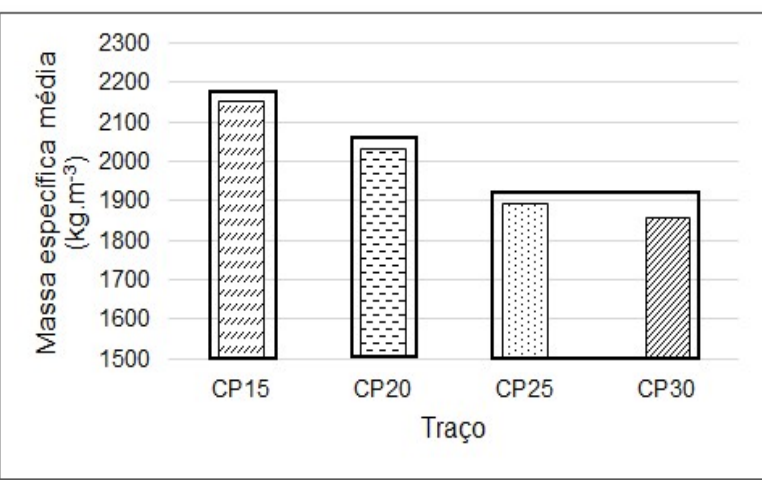

B)

Figura 4: A) Massa específica média dos concretos permeáveis, B) Comparação das médias de massa específica Onde: CP15 = Corpo de prova com índice de vazios de 15\%; CP20 = Corpo de prova com índice de vazios de $20 \%$; $\mathrm{CP} 25$ = Corpo de prova com índice de vazios de 25\%; CP30 = Corpo de prova com índice de vazios de 30\%

A resistência a compressão média dos concretos permeáveis atingiu valores que variaram de um mínimo de 13,6 MPa, para o CP30, ao máximo de 21,6 MPa, para o CP15 (Figura 5 A). A resistência a compressão média do traço CP15 não apresentou diferença significativa em relação a resistência a compressão média do traço CP20, porém ambos os traços CP15 e CP20 apresentaram diferença significativa para os traços CP25 e CP30, que entre si não tiveram diferença significativa (Figura 5 B).

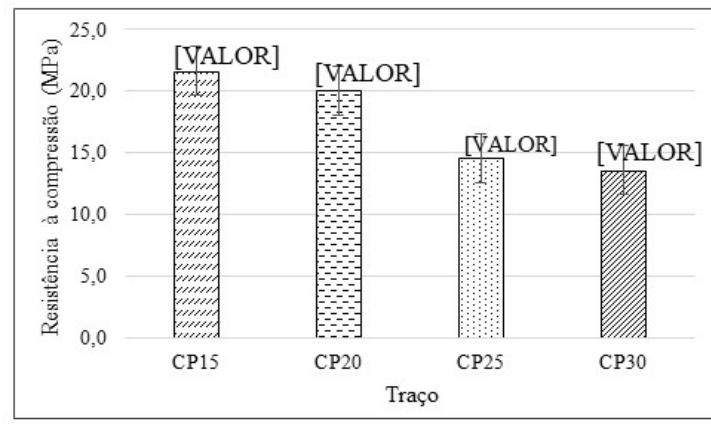

A)

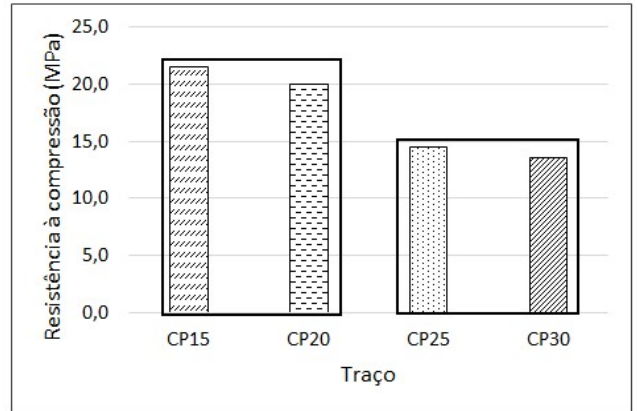

B)

Figura 5: A) Resistência a compressão média, B) Comparação das médias de resistência a compressão dos concretos permeáveis. Onde: $\mathrm{CP} 15$ = Corpo de prova com índice de vazios de 15\%; CP20 = Corpo de prova com índice de vazios de 20\%; CP25 = Corpo de prova com índice de vazios de 25\%; CP30 = Corpo de prova com índice de vazios de $30 \%$

Assim como no concreto convencional, o concreto permeável tem vários fatores que impactam em sua resistência mecânica, podendo-se destacar a porosidade, relação a/c, adensamento e o tipo e tamanho 
dos agregados utilizados (NEVILLE et al., 2013; NGOHPOK et al. 2018), assim como as propriedades da argamassa e a zona de transição entre os agregados e a argamassa (VANCURA et al. 2011; AGAR et al. 2013). A textura e a angularidade dos agregados contribuem significativamente para o desempenho da resistência mecânica do concreto permeável (LIANet al., 2011). Diversos estudos comprovam que a utilização de agregados com granulometria abaixo de $12,5 \mathrm{~mm}$ proporciona maior resistência ao concreto permeável (ZHONGE et al., 2015; IBRAHIM et al., 2014; LIAN et; ZHUGE, 2011), fato esse que vem de encontro com a brita 0 utilizada nessa pesquisa.

No que diz respeito à já bem estabelecida influência de que o aumento da porosidade implica no decréscimo da resistência mecânica do concreto permeável (COSTA, 2019; SATA et al., 2013), observa-se que os traços CP15 e CP20, que correspondem a menores porosidades (Figura 6) foram os que tiveram melhor desempenho na resistência mecânica.

A resistência à tração na flexão também apresentou resultados que podem ser considerados muito bons de acordo com a literatura estudada, uma vez que a tração na flexão de concretos permeáveis variou de 0,3 a 9,7 MPa (IBRAHIM et al., 2014; TORRES et at., 2015; COSIC et al., 2015), enquanto neste estudo seu valor médio variou entre 4,5 e 4,0 MPa para o índice de vazios do concreto permeável variando de 15 a $30 \%$, respectivamente (Figura 6).

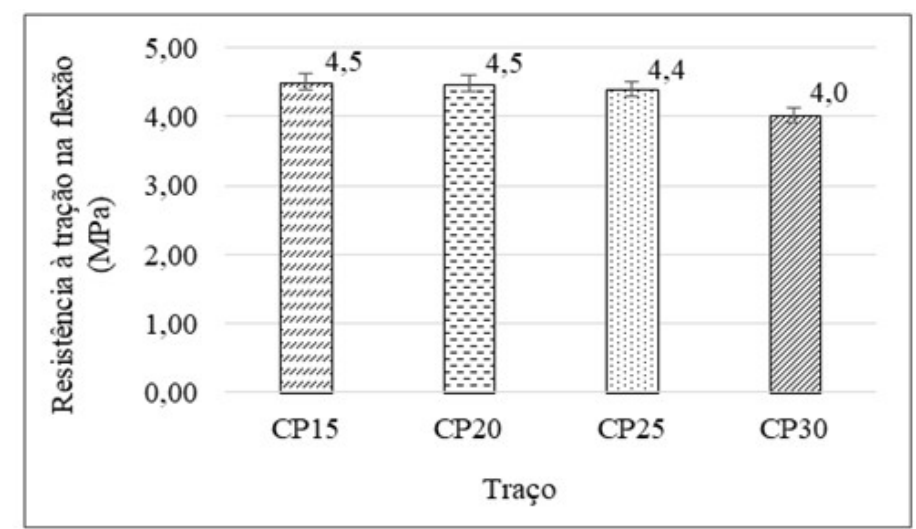

Figura 6: Resistência à tração média

Onde: $\mathrm{CP} 15$ = Corpo de prova com índice de vazios de 15\%; CP20 = Corpo de prova com índice de vazios de 20\%; CP25 = Corpo de prova com índice de vazios de 25\%; CP30 = Corpo de prova com índice de vazios de 30\%

É importante ressaltar que os resultados, independentemente do índice de vazios do concreto permeável adotado, superaram o limite estabelecido pela norma NBR 16416 (ABNT, 2015) que exige a resistência mínima para este parâmetro de $2 \mathrm{MPa}$ quando o concreto permeável for utilizado em forma de placas na condição de solicitação de tráfego leve. Ao se observar o resultado de resistência à compressão, percebe-se que a resistência à tração na flexão não apresenta a mesma tendência em função da variação do índice de vazios do concreto permeável, pois o tratamento estatístico das médias de resistência a compressão mostrou diferença significativa entre os resultados, não havendo, porém, diferença significativa quando se avaliou a resistência a tração na flexão.

Os valores de resistência à tração na flexão obtidos correspondem entre 21 e $30 \%$ dos valores de resistência a compressão, relação maior que a encontrada em alguns estudos na literatura sobre concreto permeável que variam entre 12 e 17\% (LIAN et al., 2010; IBRAHIM et al., 2014; TORRES et al., 2015; COSIC 
et al., 2015; RANGELOV et al., 2016). Segundo Neville et al. (2013), no ensaio de resistência a tração na flexão há maior influência da forma dos agregados, sendo que agregados britados e menos arredondados, como os utilizados neste trabalho, resultam em uma resistência mais elevada.

É notável a variação da condutividade térmica do concreto permeável em função da variação de sua porosidade apresentando valores médios que diminuíram de 0,55 , a $0,44 \mathrm{~W} \cdot \mathrm{m}^{-1} \cdot \mathrm{K}^{-1}$ à medida que o índice de vazios (e consequente porosidade) aumentou de 15 a 30\% (Figura 7 A). Verifica-se que existe diferença estatisticamente significativa entre a condutividade térmica dos concretos permeáveis de índice de vazios de 15 e $25 \%$, bem como entre os concretos permeáveis com índice de vazios de 15 e $30 \%$, sendo que o valor da condutividade térmica do concreto permeável com um índice de vazios de $20 \%$ não difere de nenhum outro dos concretos permeáveis com 15, 25 ou 30\% de índice de vazios.

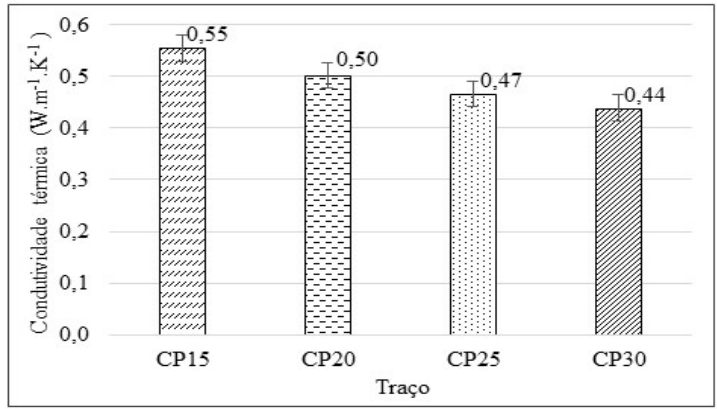

A)

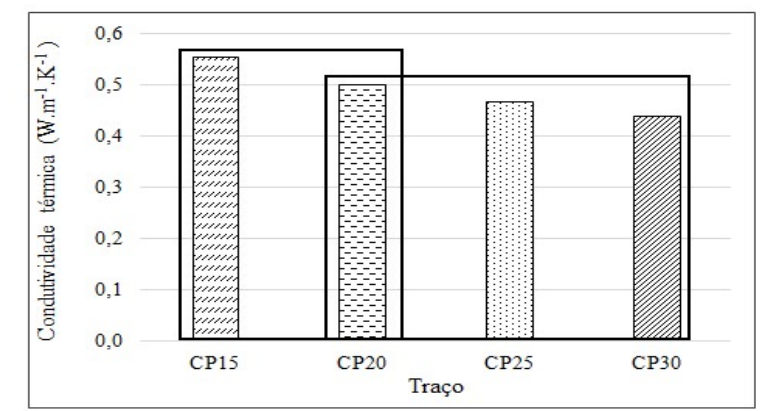

B)

Figura 7: A) Valores médios de condutividade térmica dos concretos permeáveis, B) Comparação das médias de condutividade térmica dos concretos permeáveis. Onde: $\mathrm{CP} 15=$ Corpo de prova com índice de vazios de $15 \% ; \mathrm{CP} 20=$ Corpo de prova com índice de vazios de 20\%; CP25 = Corpo de prova com índice de vazios de 25\%; CP30 = Corpo de prova com índice de vazios de $30 \%$

A condutividade térmica está relacionada com a fração do volume tipo e quantidade dos seus componentes, isto é, o cimento, os agregados, a água e como cada componente está distribuído no interior do concreto permeável, assim como de sua porosidade (SUN et al., 2017, NGOHPOK et al., 2018; CHINDAPRASIRT et al., 2014).

O cimento tem condutividade térmica que varia de 1,1 a $1,6 \mathrm{~W} \cdot \mathrm{m}^{-1} \cdot \mathrm{K}^{-1}$, e os agregados comumente utilizados em concretos de 1,0 até 4,2 W. $\mathrm{m}^{-1} \cdot \mathrm{K}^{-1}$, enquanto condutividade térmica da água e do ar é próxima de 0,58 e 0,26 W.m. $\mathrm{m}^{-1} \cdot \mathrm{K}^{-1}$ respectivamente, podendo variar devido a temperatura (ALVES, 2014; Laboratório de Propriedades Termofísicas e Prototipação de São Paulo, 2020). De acordo com Sun et al. (2017) outro fator que influencia na condutividade térmica do concreto permeável é a utilização de aditivos, porém depende do tipo de aditivo e da quantidade aplicada no traço.

Seguindo este contexto podem-se citar os trabalhos desenvolvidos por Sun et al. (2017) e Chindaprasirt et al. (2014) para evidenciar a influência dos materiais na confecção do concreto permeável, sendo que esses autores utilizaram resíduos leves como substituição parcial do agregado graúdo. Devido ao uso desses agregados leves, ambos os estudos obtiveram concretos permeáveis com baixas massas específicas, resultando também em bons resultados para a condutividade térmica que variou de 0,09 a 0,13 W. $\mathrm{m}^{-1} \cdot \mathrm{K}^{-1}$ (SUN et al., 2017) e de 0,20 a $0,22 \mathrm{~W} \cdot \mathrm{m}^{-1} \cdot \mathrm{K}^{-1}$ (CHINDAPRASIRT et al., 2014), porém dentro da literatura citada, os resultados de resistência a compressão foram os menores, variando entre 4,00 e 4,37 e 
entre 2,4 e 4,1 MPa para Sun et al. (2017) e Chindaprasirt et al. (2014), respectivamente. Chen et al. (2019) ressaltam a importância do equilíbrio entre a resistência mecânica e a condutividade térmica do concreto permeável para que ele possa ganhar mercado como um material eficaz na construção civil.

Fica ainda mais acentuada a importância da porosidade no concreto permeável, para fins de condutividade térmica, quando se compara os valores de condutividade térmica do concreto permeável com o concreto convencional, que segundo a NBR 15220 (ABNT, 2005) é próximo de 1,75 W.m $\mathrm{m}^{-1} \cdot \mathrm{K}^{-1}$, se a sua massa específica assumir valores entre 2200 e 2400 kg.m-3. A maior condutividade térmica do concreto permeável da presente pesquisa foi de $0,55 \mathrm{~W} \cdot \mathrm{m}^{-1} \cdot \mathrm{K}^{-1}$, correspondendo a uma condutividade térmica aproximadamente 3 vezes menor do que a do concreto convencional.

A relação inversamente proporcional entre a porosidade e a condutividade térmica do concreto permeável é expressa através de uma regressão linear (Figura 8), apresentando-se nesse mesmo gráfico os resultados obtidos por Nassari et al. (2017), observando-se que os resultados apresentam tendência similar. Como se pode observar, o coeficiente de determinação $\left(R^{2}\right)$ do presente trabalho demonstra um melhor ajuste dos dados à regressão linear, evidenciando pouca dispersão dos resultados, mostrando uma ótima linearidade entre a variação da condutividade térmica e os resultados de porosidade.

Nassari et al. (2017) atribuem essa tendência à baixa condutividade térmica do ar em comparação com os outros componentes do concreto permeável, assim, quanto maior concentração de ar nas placas, menor é a condutividade térmica do material. Ainda de acordo com esses autores, os resultados implicam que a elevada porosidade do concreto permeável reduz substancialmente a quantidade de calor transferido por condução, contribuindo para um melhor isolamento térmico, ou seja, a elevada porosidade e o tamanho relativamente grande dos espaços vazios dificultam a transferência de calor entre as faces internas e externas do corpo de concreto permeável.

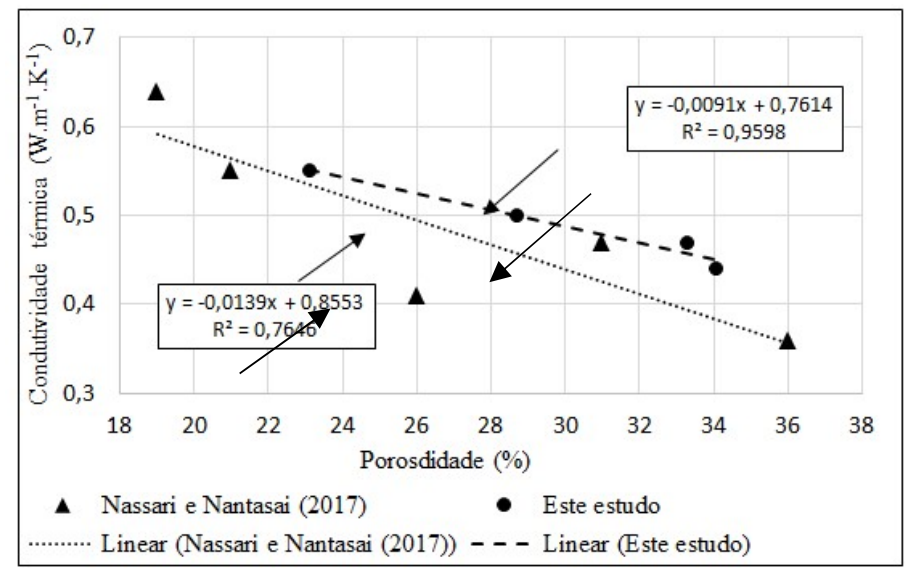

Figura 8: Relação da condutividade térmica com a porosidade total do concreto permeável.

A linha de tendência dos resultados de Nassari et al. (2017) apresenta uma inclinação mais acentuada, o que implica que a variação da condutividade térmica é maior à medida que varia a porosidade. Uma das possíveis explicações para essa maior variação da condutividade térmica pode estar relacionada aos diferentes materiais utilizados nos trabalhos, pois segundo relatam Wu et al. (2017) cada material tem diferente comportamento térmico, que influencia ou não com representatividade nas 
propriedades térmicas. Além dos diferentes materiais utilizados nas pesquisas, outros fatores que podem influenciar na dispersão dos resultados de condutividade térmica são a umidade e irregularidade da amostra utilizada para o ensaio e a calibração do equipamento (LI et al., 2017; PACHLA, 2017).

\section{CONCLUSÕES}

A variação da porosidade no concreto permeável tem grande importância e influência em suas características mecânicas, hidráulicas e térmicas, sendo que, apenas os resultados de resistência a tração na flexão não apresentaram diferença significativa quando se variou a porosidade. No ensaio de resistência a compressão foi possível atingir um valor médio acima de $20 \mathrm{MPa}$ para os traços com menores índices de vazios teórico de 15 e $20 \%$ (porosidade total de 23,1 e 28,9\%, respectivamente) enquanto a resistência a tração na flexão de todos os quatro traços (porosidade total compreendida em um intervalo de 23,1 a $34,0 \%$ ) alcançou com folga o limite solicitado pela norma brasileira, de $2 \mathrm{MPa}$.

O menor resultado de coeficiente de permeabilidade atingido foi de $8,24 \mathrm{~mm} . \mathrm{s}^{-1}$, para o concreto permeável dosado com um índice de vazios de $15 \%$ (porosidade total de $23,1 \%$ ), sendo esse valor muito superior ao mínimo exigido pela norma NBR 16416 (ABNT, 2015) de $1 \mathrm{~mm} \cdot \mathrm{s}^{-1}$.

Existe um limite físico de porosidade que o concreto permeável pode atingir, ou seja, conforme se aumenta o índice de vazios, a tendência é de que haja uma homogeneização nos resultados de porosidade (e índice de vazios), como pode ter acontecido para os índices de vazios acima de $25 \%$. Existe um limite físico de porosidade que o concreto permeável pode atingir, ou seja, conforme se aumenta o índice de vazios (teórico na dosagem), a tendência da porosidade total (e do índice de vazios) do concreto permeável executado tende à um valor máximo, como para os índices de vazios acima de $25 \%$.

A porosidade e a condutividade térmica do concreto permeável são inversamente proporcional, cuja tendência é expressa por uma correlação linear com um ótimo ajuste. Todos os quatro traços (porosidade total compreendida em um intervalo de 23,1 a 34,0\%) apresentaram bons resultados de condutividade térmica, podendo ser aplicado na construção civil como um material alternativo para fins da melhora do conforto térmico, inclusive levantando a hipótese de utilizá-lo como material de alvenaria, considerando que os traços com menor porosidades atingiram resistência mecânica acima de $20 \mathrm{MPa}$.

Considerando que o traço CP20 não tem diferença estatisticamente significativa de resistência a compressão com o CP15 que atingiu a maior resistência mecânica e não tem diferença significativa de condutividade térmica com o CP 30 que apresentou o melhor resultado de condutividade térmica, ele, o traço CP20, se apresenta como o mais indicado para ser empregado, devido principalmente a sua relação entre resistência mecânica e condutividade térmica ser mais equilibrada quando comparado aos demais traços.

\section{REFERÊNCIAS}

AMERICAN SOCIETY FOR TESTING AND MATERIALS. C518: Standard Test Method for Steady-State Thermal Transmission Properties by Means of the Heat Flow Meter Apparatus. Pennsylvania, 2015.
AMERICAN SOCIETY FOR TESTING AND MATERIALS. C1754: Standard Test Method for Density and Void Content of Hardened Pervious Concrete. Pennsylvania, 2012.

ABNT. Associação Brasileira de Normas Técnicas. NBR NM 
30: Agregado miúdo: Determinação da absorção de água. Rio de Janeiro, 2001.

ABNT. Associação Brasileira de Normas Técnicas. NBR NM 45: Agregados: Determinação da massa unitária e do volume de vazios. Rio de Janeiro, 2006.

ABNT. Associação Brasileira de Normas Técnicas. NBR NM 52: Agregado miúdo: Determinação da massa específica e massa específica aparente. Rio de Janeiro, 2009.

ABNT. Associação Brasileira de Normas Técnicas. NBR NM 53: Agregado graúdo: Determinação da massa específica, massa específica aparente e absorção de água. Rio de Janeiro, 2009

ABNT. Associação Brasileira de Normas Técnicas. NBR NM 248: Agregados: Determinação da composição granulométrica. Rio de Janeiro, 2003.

ABNT. Associação Brasileira de Normas Técnicas. NBR 5739: Concreto: Ensaio de compressão de corpos cilíndricos. Rio de Janeiro, 2018

ABNT. Associação Brasileira de Normas Técnicas. NBR 7211: Agregados para concreto: Especificação. Rio de Janeiro, 2009.

ABNT. Associação Brasileira de Normas Técnicas. NBR 7680: Concreto: Extração, preparo, ensaio e análise de testemunhos de estruturas de concreto. Rio de Janeiro, 2015.

ABNT. Associação Brasileira de Normas Técnicas. NBR 12142: Concreto: Determinação da resistência a tração na flexão em corpos de prova prismáticos. Rio de Janeiro, 2010.

ABNT. Associação Brasileira de Normas Técnicas. NBR 15220: Desempenho térmico em edificações. Rio de Janeiro, 66 p. 2005.

ABNT. Associação Brasileira de Normas Técnicas. NBR 16416: pavimentos permeáveis de concreto: requisitos e procedimentos. Rio de Janeiro, 2015.

AGAR, A. S.; WEERHEIJM J.; SCHLANGEN, E., VAN BREUGEL, K.. Investigating porous concrete with improved strength: Testing at different scales. Construction and Building Materials, v.41, p.480-490, 2013. DOI: https://doi.org/10.1016/j.conbuildmat.2012.12.040

ALVES, L. A.. Análise acoplada de temperatura e umidade no concreto em altas temperaturas. Dissertação (Mestrado em Engenharia Civil) - Universidade Federal do Rio de Janeiro, Rio de Janeiro, 2014.

ARENAS, E. S.. Desempenho termo energético de paredes de blocos de resíduos da construção civil. Dissertação (Mestrado em Engenharia Civil) - Universidade Federal de São Paulo, 2013.

BEZERRA, L. A. C.. Análise do desempenho térmico de sistema construtivo de concreto com EPS como agregado graúdo. Dissertação (Mestrado em Engenharia Mecânica) Universidade Federal do Rio Grande do Norte, Natal, 2003.

LABORATÓRIO DE PROPRIEDADES TERMOFÍSICAS E PROTOTIPAÇÃO. Tabela de condutividade térmica de materiais de construção. São Paulo, 2020.
BRASIL. Painel Brasileiro De Mudanças Climáticas. Contribuição do Grupo de Trabalho 3 ao Primeiro Relatório de Avaliação Nacional do Painel Brasileiro de Mudanças Climáticas. Rio de Janeiro, 2013.

BRASIL. Painel Brasileiro De Mudanças Climáticas. Contribuição do Grupo de Trabalho 1 ao Primeiro Relatório de Avaliação Nacional do Painel Brasileiro de Mudanças Climáticas. Rio de Janeiro, 2013.

BRASIL. Painel Brasileiro De Mudanças Climáticas. Contribuição do Grupo de Trabalho 2 ao Primeiro Relatório de Avaliação Nacional do Painel Brasileiro de Mudanças Climáticas. Rio de Janeiro, 2013.

CHEN, J.. Analysis of thermal conductivity of porous concrete using laboratory measurements and microstructure models. Construction and Building Materials, v.218, p.90-98, 2019. DOI: https://doi.org/10.1016/j.conbuildmat.2019.05.120

CHINDAPRASIRT, P.; NAUKLONG, P.; ZAETANG, W.; SUTUMNONGTOKUL, P.; SATA, T.. Mechanical and Thermal Properties of Recycling Lightweight pervious Concrete. Research Article: Civil Engineering, 2014. DOI: https://doi.org/10.1007/s13369-014-1563-z

ĆOSIĆ, K.; KORAT, L.; DUCMAN, V.; NETINGER, I.. Influence of aggregate type and size on properties of pervious concrete. Construction and Building Materials, v.78, n.8, p.69-76, 2015. https://doi.org/10.1016/j.conbuildmat.2014.12.073

COSTA, F. B. P.. Análise e desenvolvimento de misturas de concreto permeável para aplicação em pavimentação. Tese (Doutorado em Engenharia civil) - Universidade Federal do Rio Grande do Sul, Porto Alegre, 2019.

CURVO, F. O.. Estudo da drenabilidade de calçadas experimentais em concreto permeável no campus Armando Salles de Oliveira da USP. Dissertação (Mestrado em engenharia de transportes) - Escola Politécnica de São Paulo, São Paulo, 2017.

EPA - U.S. Environmental Protection Agency. Reducing Urban Heat Islands: Compendium of Strategies-Cool Pavements. Washington: Environmental Protection Agency, 2008.

GALUSIC, B.. Ilhas de calor urbanas em São Carlos, SP e os impactos da permeabilidade dos revestimentos urbanos horizontais. Dissertação (Mestrado em Arquitetura e Urbanismo) - Universidade de São Paulo, São Carlos, 2019.

GARTLAND, L.. Ilhas de calor: como mitigar zonas de calor em áreas urbanas. Dissertação (Mestrado em Física Ambiental) - Universidade Federal de Mato Grosso, Dourados, 2010.

HASELBACH, D.; ASCE. M.; UNIV, W, S.. Special Issue on Sustainability Engineering Education: Keeping Up with the World. Journal of Professional Issues in Engineering Education and Practice, v.141, n.2, p.91-103, 2015. DOI: https://doi.org/10.1061/(ASCE)EI.1943-5541.0000237

KALYANI, N. T.; DHOBLE.. Organic light emitting diodes: Energy saving lighting technology - A review. Renawable and Sustainable Energy Reviews, v.16, p.2629-2723, 2012. DOI: https://doi.org/10.1016/j.rser.2012.02.021 
IBRAHIM, A.; MAHMOUD, E.; YAMIN, M.; PATIBANDLAC, V.; C.. Experimental study on Portland cement pervious concrete mechanical and hydrological properties. Construction and Building Materials, v.50, p.524-529, 2014. DOI: https://doi.org/10.1016/j.conbuildmat.2013.09.022

KIA, A.; WONG, H. S.; CHEESEMAN, C. R.. Clogging in permeable concrete: a review. Journal of Environmental Management, v.1993 p.221-233, 2017. DOI: https://doi.org/10.1016/i.jenvman.2017.02.018

LI, J.; JONES, D.; WU, R.; HARVEY, J.. Development and HVS validation of design tables for permeable interlocking concrete pavimente: final report. Grant Agreement, 2009.

LIAN, C.; ZHUGE, Y.. Optimum mix design of enhanced permeable concrete - An experimental investigation. Construction and Building Materials, v.24, p.2664-2671, 2011. https://doi.org/10.1016/j.conbuildmat.2010.04.057

MATI-BAOUCHE, N.; BAYNAST, H.; SUN, S.. Polysaccharidic binders for the conception of an insulating agro-composite. Results in Materials, v.78, p.68-79. 2014. DOI: https://doi.org/10.1016/j.compositesa.2015.08.006

NASSARI, S.; NANTASAI, B.. Thermal Conductivity of Pervious Concrete for Various Porosities. ACI Materials Jounal, v.114, p.265-271. 2017. DOI: http://doi.org/10.14359/51689492

NEMATOLLAHI, B.; RANADE, R.; SANJAYAN, J.; RAMAKRISHNSN, S.. Thermal mechanical properties of sustainable lightweight strain hardening geopolymer composites. Swinburne University of Technology, p.55-64, 2017. DOI: http://dx.doi.org/10.1016/j.acme.2016.08.002

NEVILLE, A. M.; BROOKS, J. J.. Tecnologia do concreto. 2 ed. Porto Alegre: Bookman, 2013.

RAIMBAULT, G.; ANDRIEU, H.; BERTHIER, E.; JOANNIS, C.; LEGRET, M.. Infiltration des eaux pluviales à travers les surfaces urbaines - Des revêtementes imperméables aux structures-réservoirs. Bulletin des Laboratoires des Ponts et Cheussées, v.238, p.39-50, 2002.

RANGELOV, M.; NASSIRI, S.; CHEN, Z.; RUSSEL, M.; UHLMEYER, J.. Quality evaluation tests for pervious concrete pavements' placement. International Journal of Pavement Research and Technology, v.10, n.3, p.245-253, 2016. https://doi.org/10.1016/i.ijprt.2017.01.007

NGOHPOK, C.; SATA, V.; SATIENNAM, T.; KLUNGGBONGRONK, P.; CHINDRAPASIRT, P.. Mechanical Properties, Thermal Conductivity, and Sound Absorption of Pervious Concrete Containing Recycled Concrete and Bottom Ash Aggregates. Structural Engineering, v.22, p.1369-1376, 2018. https://doi.org/10.1007/s12205-017-0144-6

NGUYEN, D. H.. A modified method for the design of pervious concrete mix. Construcion and Building of pervious, v.73, p.271-282, 2014. DOI:

https://doi.org/10.1016/j.conbuildmat.2014.09.088

PACHLA, E. C.. Desempenho térmico-acústico-mecânicodurabilidade de compósitos de matriz cimentícia com reduzida massa específica reforçados por casca de palha de arroz. Dissertação (Mestrado em Engenharia Mecânica ) Universidade Federal do Pampa, Alegrete, 2017.

SALAO, E. T.. Estudo das características do solo cimento para o desempenho térmico das edificações brasileiras na zona climática bioclimática 6. Dissertação (Mestrado em Eficiência e Sustentabilidade) - Universidade Federal de Mato Grosso, Campo Grande, 2013.

SANTAMOURIS, M.. Using cool pavements as a mitigation strategy to fight urban heat island - A review of the actual developments. Renewable and Sustainable Energy Reviews. v.26, p.224-240, 2013. DOI: https://doi.org/10.1016/j.rser.2013.05.047

SATA, V.; WONGSA, A.; CHINDAPRASIRT, P.. Properties of pervious geopolymer concrete using recycled aggregates. Constr. Build. Mater, v.42, p.33-39, 2013. DOI: https://doi.org/10.1016/j.conbuildmat.2012.12.046

SOUZA, C. R.. Avaliação térmico de vedações em blocos de concreto produzidos com lã de rocha. Dissertação (Mestrado em Engenharia Ambiental Urbana) Universidade Federal da Bahia, Salvador, 2017.

SUN, Y.; GAO, P.; GENG, F.; LI, H.; ZHANG, L.; LIU, H.. Thermal conductivity and mechanical properties of porous concrete materials. Materials Letters, v.209, p.349-352, 2017. DOI: https://doi.org/10.1016/i.matlet.2017.08.046

TENNIS, P. D.; LEMMING, M. L.; AKERS, D. J.. Porous concrete paviments. Cement and Concrete, v.9, p.45-53, 2004.

TORRES, A.; HU, J.; RAMOS, A.. The effect of the cementitious paste thickness on the performance of pervious concrete. Construction and Building Materials, v. 95, p.850-859, 2015. DOI: https://doi.org/10.1016/i.conbuildmat.2015.07.187

VANCURA, M.; MACDONALD, K.; KHAZANIVICH, L.. Microscopic analysis of paste and aggredate distresses in pérvios concrete in a wet, hard climate. Science Direct, v.15, p.49-55, 2011. DOI: https://doi.org/10.1590/s198341952017000400004

WU, H.; SUN, B.; LIU, Z.; YIN, J.. Laboratory-simulated investigation on thermalbehaviours of permeable concrete pavements. Road Mater. Pavement, v.8, n.3, p.104-115, 2017. DOI:

http://dx.doi.org/10.1080/14680629.2017.1329865

WANDERLEY, G. V.. Produção de material para enchimento leve de elementos das edificações a partir da mistura de geopolímero com argila expandida. Dissertação (Mestrado em engenharia civil e ambiental) - Universidade Federal de Pernambuco, Curuaru, 2018.

ZHONG, R.; WILLE, K.. Material design and characterization of high performance pervious concrete. Construction and Building Materials, v.98, p.51-60, 2015. DOI: http://dx.doi.org/10.1016/j.enpol.2015.03.016

A CBPC - Companhia Brasileira de Produção Científica (CNPJ: 11.221.422/0001-03) detém os direitos materiais desta publicação. Os direitos referem-se à publicação do trabalho em qualquer parte do mundo, incluindo os direitos às renocães, expansões e disseminac̃es da contribuič̃o, bem como outros direitos subsidiários. Todos os trabalhos publicados eletronicamente podera posteriormente ser publicados em coletâneas impressas sob coordenaça da Sustenere Publishing da Companhia Brasileira de Produção Científica e seus parceiros autorizados. Os (as) autores (as) preservam os direitos autorais, mas não têm permissão para a publicação da contribuição em outro meio, impresso ou digital, em português ou em tradução. 